\title{
Linx
}

Revue des linguistes de l'université Paris X Nanterre

68-69 | 2013

Corpus et apprentissage du français

\section{L'acquisition du schwa en français L1 : analyse de corpus denses d'interactions parents - enfant}

Loïc Liégeois, Inès Saddour et Damien Chabanal

\section{(2) OpenEdition}

\section{Journals}

\section{Édition électronique}

URL : http://journals.openedition.org/linx/1512

DOI : 10.4000/linx.1512

ISSN : 2118-9692

\section{Éditeur}

Presses universitaires de Paris Nanterre

\section{Édition imprimée}

Date de publication : 19 novembre 2013

Pagination : 49-68

ISSN : 0246-8743

\section{Référence électronique}

Loïc Liégeois, Inès Saddour et Damien Chabanal, «L'acquisition du schwa en français L1 : analyse de corpus denses d'interactions parents - enfant », Linx [En ligne], 68-69 | 2013, mis en ligne le 19 novembre 2015, consulté le 30 avril 2019. URL : http://journals.openedition.org/linx/1512 ; DOI : 10.4000/linx.1512 


\title{
L'acquisition du schwa en français L1 : analyse de corpus denses d'interactions parents - enfant
}

\author{
Loïc Liégeois, Clermont Université, Université Blaise Pascal, Laboratoire de \\ Recherche sur le langage \\ Inès Saddour, Université de Toulouse Jean Jaurès, Octogone-Lordat \\ Damien Chabanal, Clermont Université, Université Blaise Pascal, \\ Laboratoire de Recherche sur le langage
}

\section{Introduction}

L'utilisation des données orales dans le cadre des recherches en acquisition du langage est devenue une pratique courante à la fin du $20^{\mathrm{e}}$ siècle. Alors que les chercheurs étaient habitués à examiner des recueils de notes construits à partir des productions orales d'enfants de leur entourage proche (Parisse \& Morgenstern, 2010a, 2010b), l'évolution des technologies a engendré une multiplication des corpus de langage spontané et leur exploitation a bouleversé le champ des recherches en acquisition du langage. Dans le cadre du projet ALIPE (Acquisition de la Liaison et Interactions Parents-Enfants), nous avons collecté environ 30 heures d'interactions parents-enfants en situation naturelle (pendant les repas, le bain ou lors de situations de jeux).

Nous proposons dans cet article d'exploiter ce corpus dans le but d'étudier l'acquisition d'un phénomène de variation phonologique particulier chez le jeune enfant: l'élision du schwa dans les monosyllabes. Comme la liaison, le schwa (ou «e muet», "e caduque ») est un phénomène de sandhi du français qui a fait l'objet de 
nombreuses analyses proposées par des courants phonologiques différents, de la théorie générative linéaire (Dell, 1973, 1978) à la théorie de l'optimalité (Eychenne, 2006). Cependant, alors que le schwa est devenu une thématique de recherche privilégiée en phonétique et en phonologie, la question de son acquisition a été peu abordée (Nicoladis \& Paradis, 2011). L'étude que nous proposons a pour objectif de combler ce manque et d'analyser la variation entre réalisation et omission du schwa dans le discours parental et enfantin. En nous focalisant sur les contextes de schwas dans les monosyllabes $c e, d e, j e, l e, m e, n e, q u e, s e$ et te, nous étudierons les particularités du discours adressé à l'enfant et son influence sur l'acquisition de cette variation par le jeune locuteur.

\section{Input et acquisition d'une variable phonologique}

Le schwa est une voyelle pouvant alterner avec zéro dans un même contexte lexical en fonction de différents critères (prosodiques ou stylistiques par exemple). On distingue traditionnellement cinq contextes dans lesquels le schwa peut apparaitre : dans un monosyllabe (le train), en syllabe interne d'un polysyllabe (finalement), dans la première (monsieur) ou la dernière syllabe d'un polysyllabe (cavale) et dans le cas d'une métathèse (je viens [əзुvjẽ]). Sa nature phonétique est propre, le schwa se distingue du $[œ]$ et du [ø]. D'un point de vue phonologique, la question la plus importante repose sur le fait de savoir si le schwa est sous-jacent (Dell, 1973 ; Schane, 1968) ou épenthétique (Martinet, 1972). S'il est reconnu que le schwa est sujet à une variation régionale (Bürki et al., 2008), il apparait que son usage en régions Auvergne et Bourgogne, dont sont originaires nos sujets, ne diffère pas du français standard (Chabanal \& Durand, 2015).

Pour expliciter de manière claire notre conception de l'acquisition d'une variable phonologique, nous nous appuierons sur une métaphore de l'acquisition du langage présentée par Pierrehumbert (2001). Cette dernière compare l'acquisition d'une catégorie phonologique à l'apprentissage d'une note précise de musique. Elle explique qu'un enfant qui apprend le violoncelle produit des accords de notes variables au début, et de plus en plus précis au cours des années de pratique. L'enfant parvient donc, grâce à la pratique et à la fréquence d'écoute de la note souhaitée, à extraire l'exemplaire sonore recherché et grâce à l'entrenchment, à le stocker et l'activer comme le prototype le plus souhaitable parmi l'ensemble des sons présents dans cette même catégorie. Cette métaphore nous renseigne sur les trois principes défendus par la linguistique fondée sur l'usage, à laquelle nous souscrivons pour expliquer l'acquisition du schwa. Chronologiquement, l'enfant réalise, à partir de l'input, des notes qu'il parvient plus ou moins à reproduire. De la même manière, la production du schwa au départ semble dépendre de la mémorisation de formes non segmentées. L'enfant stockerait des morceaux de phrases ou chunks contenant ou non le «e muet». Ce stade est similaire au stade des holophrases défendu par Tomasello (2003). Dans un deuxième temps, grâce au stockage d'éléments mémorisés et à la fréquence d'exemplaires de la note «juste», l'enfant parvient à comprendre, par analogie et par raisonnement, l'ensemble des emplacements de doigts à positionner sur le manche du violoncelle pour produire le bon son et à abstraire la représentation du geste à donner. De la même manière, grâce à la fréquence de 
collocations lexicales fournies par l'input, il pourrait mémoriser des tokens dont les plus fréquents formeront des types à savoir des constructions plus générales. À l'aide de processus analogiques, ces constructions lui permettront d'acquérir le schéma d'alternance phonologique du schwa. La théorie basée sur l'usage rapportée à l'acquisition est vue de la manière suivante par Tomasello (2003). Dans le processus d'acquisition, l'enfant opère deux actions cognitives de manière simultanée. Premièrement, il extrait des occurrences sous formes d'expressions syntagmatiques non segmentées ou des éléments plus petits tels que des mots ou des morphèmes et fait le lien avec leur rôle communicatif. Deuxièmement, il repère des schémas récurrents puis construit des modèles à travers les occurrences ou parties d'occurrences ayant des structures (constructions) et fonctions communicatives similaires. Au final, cela lui permet de créer des catégories ou des constructions abstraites. Il y aurait donc, selon Tomasello, deux faces dans la construction d'une grammaire : la mémorisation d'éléments concrets du langage et de grands modèles (patterns) construits sur la base de ces mémorisations.

Au final, l'usage, la fréquence dans l'input et l'abstraction structureraient l'émergence de patterns phonologiques réguliers.

\section{Variation phonologique en discours adressé à l'enfant}

Plusieurs études attestent que le discours adressé à l'enfant (DAE) présente diverses particularités syntaxiques (Phillips, 1973 ; Rondal, 1980 ; Snow, 1972), prosodiques (Jisa \& Richaud, 1994; Garnica, 1977), phonologiques (Foulkes et al., 2005) ou phonétiques (Uther et al., 2007 ; Dilley et al., 2013) qui le différencient du discours adressé à l'adulte (DAA). Au niveau phonologique, par exemple, les liaisons variables sont davantage réalisées en DAE qu'en DAA (Liégeois et al., 2011). Quant à l'élision du schwa, elle s'avère moins fréquente en DAE, en particulier dans les substantifs, reflétant « la proéminence à la fois fonctionnelle et structurale qui leur serait assignée » (Andreassen, 2011 : 74). Par ailleurs, certaines études ont mis en évidence le rôle des caractéristiques du DAE dans l'acquisition du langage (ex. Chenu \& Jisa, 2005). En revanche, très peu d'entre elles se sont intéressées à l'acquisition du schwa. Dans son étude, Andreassen (2011) a analysé le contexte V\#Cə dans les productions enfantines et comparé le DAE et le DAA en se basant sur des corpus de données recueillies dans des contextes différents. En effet, elle a comparé le DAE récolté au cours d'interactions parents-enfants à des données du DAA extraites du projet $\mathrm{PFC}^{1}$ (Durand, Laks \& Lyche, 2002, 2009). Dans nos travaux antérieurs (Liégeois et al., 2012), nous avons apporté, à partir de l'analyse de productions orales de deux enfants en interaction avec leurs parents, un certain éclairage sur les particularités du discours adressé à l'enfant. En effet le DAE contient beaucoup moins d'élisions que le DAA et les parents semblent ajuster et moduler leur discours en fonction du développement linguistique de leur enfant. Dans cette étude, les productions d'un troisième enfant (Prune) sont analysées, ce qui permettrait d'approfondir nos résultats précédents. Par ailleurs, nous souhaitons prendre en considération le contexte syntaxique dans lequel est

\footnotetext{
${ }^{1}$ Phonologie du Français Contemporain : http://www.projet-pfc.net/.
} 
produit le monosyllabe, ce qui nous aiderait à vérifier si, comme l'a observé Andreassen (2011) pour les polysyllabes, le taux d'élision est plus faible au sein d'un groupe nominal dans le discours adressé à l'enfant. Enfin, nous nous focaliserons sur le monosyllabe «le» qui a la particularité de jouer deux fonctions dans le discours : il peut être utilisé soit comme un pronom (1) soit un déterminant (2):

1. Je le prends

\section{Je prends le ballon}

Nous faisons l'hypothèse que si l'effet de "proéminence fonctionnelle et structurale » (Andreassen, 2011) du nom est visible de par une tendance au maintien du schwa à l'intérieur de celui-ci, alors cet effet devrait également s'appliquer au déterminant qui peut l'accompagner. De ce fait, les parents devraient davantage maintenir le schwa dans des contextes nominaux, dans le but d'offrir en priorité à l'enfant des formes standard, non vernaculaires, facilitant ainsi la segmentation de l'énoncé et l'acquisition lexicale. Pour répondre à ces problématiques, nous nous appuierons sur les données extraites des trois corpus du projet ALIPE (Liégeois et al., 2014).

\section{Corpus et méthodologie}

\subsection{Corpus et acquisition du langage}

Malgré les évolutions technologiques qui permettent aujourd'hui aux chercheurs d'avoir accès à un bon nombre d'outils d'aide à la transcription, l'annotation ou l'analyse des interactions, les tâches relatives à la constitution d'un corpus de données issues d'interactions naturelles restent chronophages. Au cours du temps, les chercheurs ont été amenés à se confronter à de nouvelles problématiques concernant, par exemple, la matérialisation du signal sonore, l'enrichissement des données primaires ou le format d'encodage des données. En Sciences du langage, les travaux s'appuyant sur des analyses réalisées à partir de productions écrites ou orales constituant un corpus se sont multipliés. Bien plus qu'un simple effet de mode, cet engouement témoigne d'une redéfinition de l'objet étudié : l'observation de corpus d'énoncés produits en contexte est préférée à l'étude d'exemples construits et souvent jugés en fonction de la seule intuition du linguiste. Ce changement méthodologique est à mettre en parallèle avec l'évolution des cadres théoriques d'analyse.

Selon Laks (2011), parallèlement au développement des banques de corpus et de leurs outils d'analyse associés, la critique du modèle chomskyen a pris de l'ampleur, et ce dans les différents champs de recherche linguistique (syntaxe, sémantique ou phonologie). Ainsi, les modèles prenant davantage appui sur les «productions réelles » que sur les intuitions du sujet parlant se développent. Les théories basées sur l'usage (Barlow \& Kemmer, 2000) ou les théories exemplaristes (Bybee, 2010 ; Pierrehumbert, 2001) et les grammaires de constructions (Goldberg, 2006) prennent de l'essor en proposant des cadres se confrontant davantage à des productions attestées qu'à des exemples construits. 
En ce qui concerne les champs de recherche en phonologie et en acquisition du langage, le contexte est, semble-t-il, légèrement différent. Le courant générativiste et ses arguments contre l'utilisation des corpus ont certes influencé ces deux champs d'étude. Cependant, la nature même des recherches en phonologie et en acquisition du langage fait qu'elles entretiennent un lien indéniable avec la "performance», la production d'énoncés en situation et tout simplement l'oral. Opposant linguistique de «l'exemplum» et linguistique du «datum», Laks (2011: 5) affirme que «s'il est un domaine où la description des usages est nécessairement dominante et où le raisonnement à partir d'exemples choisis est plus que marginal, c'est bien celui de la phonologie». En effet, comment le phonologue pourrait-il décrire et analyser des phénomènes linguistiques sans procéder à un recueil des productions possibles dans la langue étudiée ? Pour tester la « grammaticalité » d’un énoncé oral, la seule introspection du phonologue ne peut suffire, celle-ci étant forcément conditionnée par sa propre expérience linguistique et « sa sensibilité sociale à la variation des usages » (Laks, 2011 : 6). En ce qui concerne l'acquisition du langage, la donne est relativement proche. Lorsque le langage de l'enfant pré-lecteur est au centre des études, il apparait difficile de se démarquer de ses productions en contexte. Ainsi, les chercheurs ont presque toujours procédé à une collecte de données, les moyens utilisés pour le recueil évoluant au fil du temps. Si les premières études scientifiques réalisées à partir de recueils d'interactions en situation naturelle (Darwin, 1877 ; Taine, 1876, 1877) se sont basées sur des prises de notes «à la volée » (parental diaries), l'évolution des technologies a engendré un nombre croissant d'études menées à partir de corpus oraux ou multimodaux (Behrens, 2008). La nature même des données exploitées s'est diversifiée. Alors que les principaux sujets observés par les chercheurs étaient souvent leurs propres enfants, l'hétérogénéité des données s'est développée d'un point de vue sociolinguistique. Aujourd'hui, les milieux sociaux des sujets enregistrés sont variés, ouvrant la voie à des études sociolinguistiques de l'acquisition de certains phénomènes langagiers (Chabanal, 2003). Cette diversification, mêlée à la multiplication des données accessibles, permet en outre une prise en compte des variations inter-locuteurs et intra-locuteurs et des phénomènes variant et invariant du processus d'acquisition du langage.

\subsection{Le corpus ALIPE : Méthodologie de recueil et de structuration}

Les analyses présentées dans cet article sont basées sur les données issues des corpus du projet ALIPE (Liégeois et al., 2014). Plus spécifiquement, il s'agit de corpus oraux recueillis en situation naturelle d'interaction entre trois enfants et leurs parents respectifs. Les trois sujets de cette étude sont des enfants premiers nés au sein de familles biparentales ayant le français comme seule langue maternelle. Lors du recueil des corpus, Salomé et Prune étaient filles uniques alors que Baptiste était l'aîné d'un deuxième garçon, né quelques mois avant la première session d'enregistrement. Les six parents enregistrés ont un indice de position socio-économique les plaçant dans la classe moyenne ou moyenne-supérieure en référence à la méthodologie de calcul de l'indice de position socio-économique définie par Genoud (2011). En ce qui concerne la scolarisation des enfants, seule Salomé n'était pas encore scolarisée lors du premier temps d'enregistrement mais gardée au domicile d'une assistante maternelle. Lors du second, elle avait intégré, comme Baptiste et Prune, une école maternelle publique. 
Pendant une semaine, les parents avaient pour consigne de déclencher l'enregistreur numérique équipé d'un microphone omnidirectionnel intégré entre trente minutes et une heure par jour. De cette façon, nous avons minimisé les biais qui auraient pu être engendrés par l'intrusion d'un observateur inconnu de l'enfant à son domicile (Tomasello \& Stahl, 2004). L’unique consigne donnée aux parents était d'enregistrer leur enfant pendant le bain, le repas, des séances de jeux ou dans toute autre condition favorisant les interactions parents-enfant ${ }^{2}$. Ainsi, en répétant le même protocole entre sept et huit mois plus tard, nous avons été en mesure d'obtenir des corpus relativement denses recueillis en deux temps (T1 et T2) - voir le Tableau 1. Il est reconnu que l'utilisation de corpus denses permet d'obtenir un panorama des formes et des constructions que l'enfant maitrise, en compréhension comme en production tout en rendant possible l'étude de phénomènes linguistiques relativement rares (Tomasello \& Stahl, 2004). Dans notre cas, nous avons pu recueillir au total 8205 contextes de production ou d'élision du schwa dans un monosyllabe (cf. Annexe 1).

\begin{tabular}{|c|c|c|c|}
\hline \multirow{2}{*}{ Enfant } & Age & $\begin{array}{c}\text { Durée totale des } \\
\text { enregistrements }\end{array}$ & $\begin{array}{c}\text { Durée des } \\
\text { enregistrements transcrits } \\
\text { et annotés }\end{array}$ \\
\hline \multirow{2}{*}{ Salomé } & $\mathrm{T} 1: 2 ; 4 \mathrm{ans}^{3}$ & $8 \mathrm{~h} 06$ & $5 \mathrm{~h}$ \\
\cline { 2 - 4 } & $\mathrm{T} 2: 3 ; 0$ ans & $6 \mathrm{~h} 42$ & $5 \mathrm{~h}$ \\
\hline \multirow{2}{*}{ Baptiste } & $\mathrm{T} 1: 3 ; 0$ ans & $5 \mathrm{~h} 30$ & $5 \mathrm{~h} 30$ \\
\cline { 2 - 4 } & $\mathrm{T} 2: 3 ; 7$ ans & $4 \mathrm{~h} 23$ & $4 \mathrm{~h} 23$ \\
\hline \multirow{2}{*}{ Prune } & $\mathrm{T} 1: 3 ; 4$ ans & $8 \mathrm{~h} 34$ & $5 \mathrm{~h}$ \\
\cline { 2 - 4 } & $\mathrm{T} 2: 4 ; 0$ ans & $2 \mathrm{~h} 02$ & $2 \mathrm{~h} 02$ \\
\hline
\end{tabular}

Tableau 1 : Durées des enregistrements récoltés et transcrits

\subsection{Transcription et structuration du corpus}

Les méthodes de transcription et d'annotation de données orales sont diverses et peuvent varier en fonction de plusieurs critères, impactant ainsi le temps passé à structurer les données. Par exemple le transcripteur peut, selon ses objectifs de recherche, utiliser une méthode de transcription plus ou moins détaillée, allant de la transcription orthographique à la transcription acoustique-phonétique (DelaisRoussarie, 2004). Si on estime que le temps passé à transcrire et annoter une heure de conversation peut atteindre vingt heures (Behrens, 2008b ; Parisse \& Morgenstern, 2010a), la quantité de phénomènes linguistiques et paralinguistiques à annoter peut faire varier ce temps moyen. Dans le but de faciliter les analyses lexicales, nous avons opté dans le cadre du projet ALIPE pour une transcription orthographique. En

\footnotetext{
2 Dans la majorité des cas, les parents ont choisi de procéder aux enregistrements lors des repas. Notons que les moments de lecture ont été écartés afin de ne pas prendre en compte dans les données les énoncés lus et les nombreuses reprises que provoque ce contexte particulier d'interaction.

${ }^{3}$ Lire 2 ans et 4 mois. Les âges des enfants sont précisés au format ANNÉE;MOIS;(JOUR).
} 
revanche, nous nous sommes focalisés sur certains phénomènes phonologiques, leur attribuant un jeu d'annotation spécifique.

Pour la transcription et l'annotation des données du projet ALIPE, nous avons décidé d'utiliser le langage de balisage XML ("eXtensible Markup Language») en définissant nous-mêmes un schéma de structuration (le format XML-ALIPE). Le choix d'utiliser le langage XML s'est imposé à nous au regard de son expressivité et des possibilités qu'il offre dans un but de standardisation en vue de l'interopérabilité et du partage des corpus langagiers (Liégeois, 2013). Ainsi, au moyen d'une série de programmes de transformation rédigés en langages Perl (Wall, Christiansen \& Orwant, 2001) et XSLT ("eXtensible Stylesheet Language Transformations»), nous avons obtenu deux versions supplémentaires de notre corpus. La première est une version au format XML-TEI («Text Encoding Initiative») mise à disposition de la communauté de chercheurs sur le serveur du Laboratoire de Recherche sur le Langage (http://lrldiffusion.univ-bpclermont.fr/). La seconde, structurée au format CHAT viendra enrichir la base de données CHILDES (MacWhinney \& Snow, 1985 ; MacWhinney, 2000) et permettra à l'équipe de recherche de mener des analyses via le logiciel associé CLAN.

\section{Analyses et Résultats}

\section{1. Élision du schwa variable dans les productions enfantines}

Afin de mener nos analyses, nous avons extrait automatiquement de notre corpus l'ensemble des monosyllabes comportant un schwa variable ainsi que leur contexte de réalisation. À la suite de cette extraction, nous avons décidé d'exclure certains contextes particuliers :

- les enchaînements de monosyllabes, comme «je le prends». En effet, la chute du schwa dans l'un des monosyllabes entraine le maintien du schwa dans l'autre. De plus, ce critère nous permet également d'exclure les contextes de répétition d'un même monosyllabe résultant par exemple d'une hésitation : «j'ai pris le le la voiture»;

- les monosyllabes présents en début ou en fin d'énoncé : «je prends la voiture » ou « vas-y prends-le ».

Ainsi, nous obtenons un ensemble de contextes de monosyllabes produits uniquement en milieu d'énoncé dont les effectifs, répartis par locuteurs, sont présentés dans l'annexe 1. Entre nos deux temps de recueil des données (T1 et T2), nos trois sujets tendent à élider davantage le schwa, ce qui corrobore les résultats que nous avions précédemment obtenus en prenant en compte l'ensemble des contextes de production des monosyllabes chez deux des trois sujets étudiés aujourd'hui (Liégeois et al., 2012). En effet, la différence de taux d'élision entre T1 et T2 se révèle significativement positive au regard d'un test de Chi2 de conformité $\left(\chi^{2}\right)$ (cf. Figure 1 et Tableau 2). 


\begin{tabular}{|c|c|c|c|c|}
\hline Enfant & $\begin{array}{l}\text { Temps de recueil et } \\
\text { âge de l'enfant }\end{array}$ & Taux d'élision & $\chi^{2}$ & $\mathbf{P}$ \\
\hline \multirow{2}{*}{ Salomé } & $\mathrm{T} 1: 2 ; 4$ ans & $10,81 \%$ & \multirow{2}{*}{$\chi^{2}=216.0006$} & \multirow{2}{*}{$\mathrm{P}<0,0001$} \\
\hline & $\mathrm{T} 2: 3 ; 0$ ans & $44,91 \%$ & & \\
\hline \multirow{2}{*}{ Baptiste } & $\mathrm{T} 1: 3 ; 0$ ans & $9,2 \%$ & \multirow{2}{*}{$\chi^{2}=6.7688$} & \multirow{2}{*}{$\mathrm{P}<0,01$} \\
\hline & T2 $: 3 ; 7$ ans & $19,02 \%$ & & \\
\hline \multirow{2}{*}{ Prune } & $\mathrm{T} 1: 3 ; 4$ ans & $44,19 \%$ & \multirow{2}{*}{$\chi^{2}=7.3637$} & \multirow{2}{*}{$\mathrm{P}<0,01$} \\
\hline & $\mathrm{T} 2: 4 ; 0$ ans & $51,9 \%$ & & \\
\hline
\end{tabular}

Tableau 2 :

Résultats du test du $\chi^{2}$ de conformité appliqué aux taux d'élision du schwa au T1 et au T2.

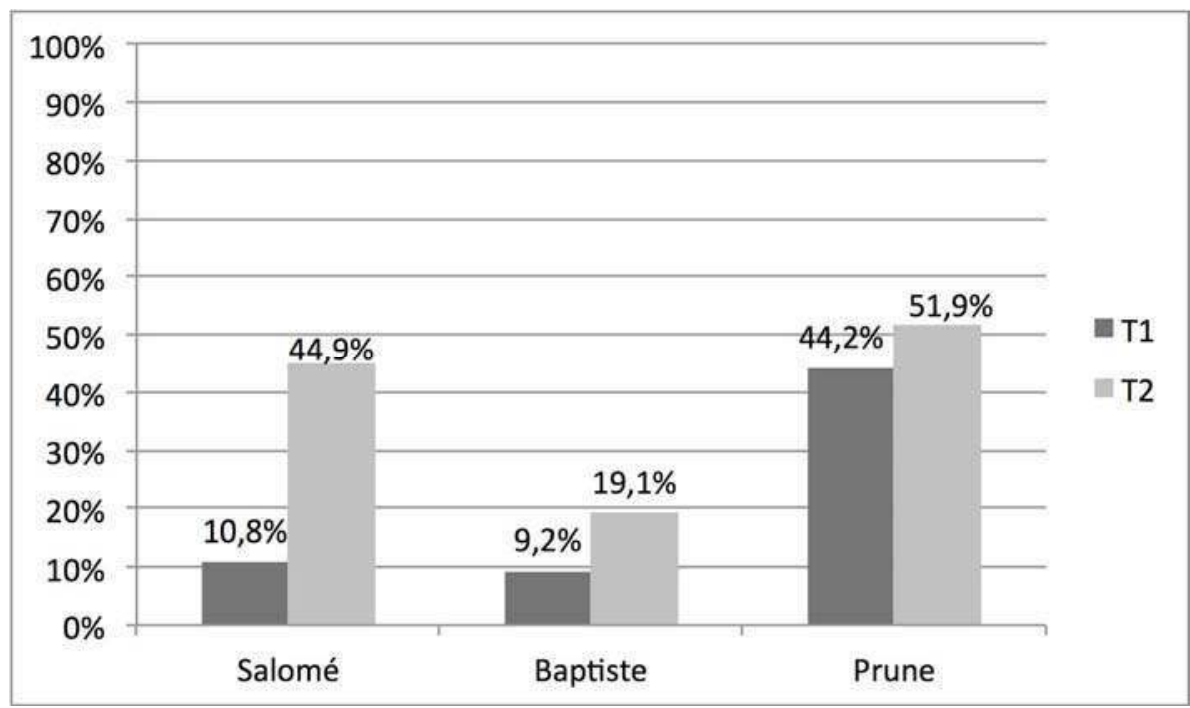

Figure 1 :

Taux d'élision dans les productions des trois enfants en fonction du temps de recueil

L'augmentation la plus nette du taux d'élision s'observe dans les productions de Salomé. En huit mois, le taux d'élision du schwa relevé dans les productions de la fillette passe d'environ $11 \%$ à 2;4 ans à un peu moins de $45 \%$ lorsqu'elle est âgée de $3 ; 0$ ans, un taux comparable à celui observé chez Prune à un âge relativement proche (3;4 ans). En comparaison, Baptiste tend à maintenir le schwa davantage que les deux fillettes, le taux d'élision relevé dans ses productions demeurant relativement faible, même au T2 lorsque ce dernier est alors âgé de 3;7 ans. Ce faible taux trouve son explication dans le développement linguistique du garçon, moins avancé que les deux fillettes : son vocabulaire est moins développé et ses énoncés sont plus courts, ce qui n'est pas surprenant au regard de la littérature existant sur l'effet du sexe de l'enfant sur la vitesse d'acquisition lexicale et sur la qualité de l'input parental (Huttenlocher et al., 1991 ; Bornstein et al., 2004), essentiellement dans les premières années. Par exemple, certains des monosyllabes relevés n'apparaissent que sporadiquement comme «ce», produit par Baptiste seulement trois fois au T2. 
Nous nous intéressons dans ce qui suit aux productions parentales afin de voir s'il y a des différences en ce qui concerne l'élision du schwa entre les deux temps de collecte de données. Par ailleurs, nous analysons les productions parentales en fonction de l'adresse du discours et en les comparant avec celles des enfants.

\section{2. Élision du schwa dans les productions parentales : effet de l'adresse du discours}

Le discours adressé à l'enfant, comme nous l'avons vu précédemment, comporte diverses spécificités en comparaison du discours adressé à un adulte. Au niveau de l'élision du schwa dans les monosyllabes, nous avions relevé dans une précédente étude que celle-ci était beaucoup moins fréquente en DAE, résultats qui corroboraient ceux obtenus par Andreassen pour les polysyllabes (Liégeois et al., 2012). Nous reportons dans la Figure 2 et le Tableau 3 les taux d'élision dans les productions parentales au T1 et au T2 en fonction de l'adresse du discours pour les parents de nos trois sujets.

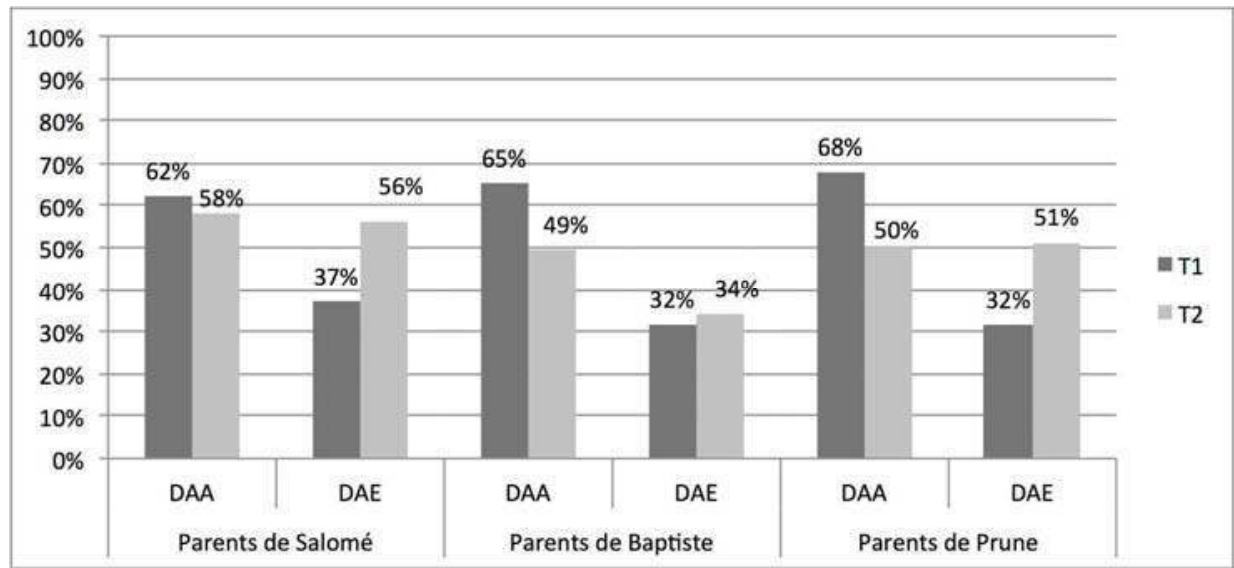

Figure 2 :

Taux d'élision du schwa dans les productions parentales en fonction du temps de recueil et de l'adresse du discours.

Nos analyses révèlent que les taux d'élision dans les productions parentales sont significativement différents en fonction de l'adresse du discours au T1 pour chacun de nos trois sujets, au regard du test du Chi2 de conformité (cf. Tableau 3). Au T2, par contre, les parents de Salomé et Prune ne semblent plus moduler leur langage en fonction de l'adresse de leur discours. Les taux d'élision des fillettes étant très proches de ceux de leurs parents respectifs, ces derniers ont ajusté leurs productions afin de correspondre au niveau linguistique de leur enfant. À l'inverse, comparé à celui observé en DAA, le taux d'élision des parents de Baptiste en DAE reste significativement plus faible au T2, le garçon ne semblant toujours pas maitriser cette variable phonologique (ceci étant déductible des faibles taux en T2 comparés à ceux des adultes). Ces 
résultats nous permettent aussi de constater que les parents ajustent et modulent leur discours en fonction des productions de leur enfant.

\begin{tabular}{|c|c|c|c|c|c|}
\hline \multirow{2}{*}{ Parents } & $\begin{array}{c}\text { Temps de } \\
\text { recueil et } \\
\text { age de } \\
\text { l'enfant }\end{array}$ & $\begin{array}{c}\text { Taux } \\
\text { d'élision en } \\
\text { DAA }\end{array}$ & $\begin{array}{c}\text { Taux } \\
\text { d'élision en } \\
\text { DAE }\end{array}$ & $\boldsymbol{\chi}^{\mathbf{2}}$ & $\mathbf{P}$ \\
\hline $\begin{array}{c}\text { Parents de } \\
\text { Salomé }\end{array}$ & $\mathrm{T} 1: 2 ; 4$ ans & $62,1 \%$ & $37,0 \%$ & Chi2=95.0865 & $\mathbf{p}<\mathbf{0 , 0 0 0 1}$ \\
\cline { 2 - 6 } & $\mathrm{T} 2: 3 ; 0$ ans & $58,2 \%$ & $56,1 \%$ & Chi2=0 & $\mathrm{p}>0,05$ \\
\hline \multirow{2}{*}{$\begin{array}{c}\text { Parents de } \\
\text { Baptiste }\end{array}$} & $\mathrm{T} 1: 3 ; 0$ ans & $65,1 \%$ & $31,7 \%$ & Chi2=75.9812 & $\mathbf{p}<\mathbf{0 , 0 0 0 1}$ \\
\cline { 2 - 6 } & $\mathrm{T} 2: 3 ; 7$ ans & $49,4 \%$ & $34,2 \%$ & Chi2=21.8028 & $\mathbf{p}<\mathbf{0 , 0 0 0 1}$ \\
\hline \multirow{2}{*}{$\begin{array}{c}\text { Parents de } \\
\text { Prune }\end{array}$} & $\mathrm{T} 1: 3 ; 4$ ans & $67,8 \%$ & $31,6 \%$ & Chi2=95.0865 & $\mathbf{p}<\mathbf{0 , 0 0 0 1}$ \\
\cline { 2 - 6 } & $\mathrm{T} 2: 4 ; 0$ ans & $50,0 \%$ & $51,2 \%$ & Chi2=0 & $\mathrm{p}>0,05$ \\
\hline
\end{tabular}

Tableau 3 :

Résultats du test du Chi2 de conformité appliqué aux taux d'élision du schwa au T1 et au T2

$\mathrm{Au}$ regard de ces résultats, nous avons décidé de détailler nos analyses en prenant en compte le contexte syntaxique de production des monosyllabes. En effet, il nous est apparu nécessaire de vérifier si la stabilisation du taux d'élision en DAE pouvait être observée en contexte verbal et en contexte nominal ou si, comme nous en faisons l'hypothèse, les parents vont continuer à davantage maintenir le schwa à l'intérieur d'un groupe nominal. Dans cet objectif, nous nous focaliserons sur le monosyllabe «le», qui peut être employé comme déterminant dans un groupe nominal ou bien comme pronom précédant ou faisant suite à un verbe. Le monosyllabe "de » nous aurait également permis de mener ce genre d'analyse mais, malheureusement, les effectifs sont trop faibles dans les productions enfantines du corpus pour pouvoir l'inclure dans cette étude.

\section{3. Élision du schwa dans les productions parentales et enfantines : effet du contexte syntaxique}

Parmi les monosyllabes sur lesquels nous nous sommes concentrés, «le» apparait comme un cas particulier. Avec un total de 2485 contextes de production relevés tous locuteurs confondus, «le» est le deuxième monosyllabe le plus employé dans notre corpus après «je» (3476 occurrences). De plus, le fait qu'il puisse être employé comme article défini ou pronom pose plusieurs problèmes.

Par exemple, nous pouvons nous demander si cette forme, sujette dans un cas comme dans l'autre au phénomène d'élision du schwa, est gérée de la même façon par les locuteurs et en particulier par les enfants. En d'autres termes, les enfants sont-ils exposés à une variation identique ou différente en fonction du statut du monosyllabe? Nous faisons l'hypothèse que, de la même façon que le schwa tend à se maintenir davantage à l'intérieur d'un nom qu'à l'intérieur d'un verbe (Andreassen, 2011), les parents réaliseront le schwa dans des proportions plus élevées lorsque «le» est employé comme un article au sein d'un groupe nominal. Une fois les contextes 
particuliers écartés (cf. Section 5.1), nous avons pu extraire 1873 contextes de production du monosyllabe «le» (cf. Tableau 4).

\begin{tabular}{|c|c|c|c|c|c|}
\hline \multirow{2}{*}{$\begin{array}{l}\text { Temps } \\
\text { de } \\
\text { recueil }\end{array}$} & \multirow[t]{2}{*}{$\begin{array}{l}\text { Locuteurs (Adresse du } \\
\text { discours) }\end{array}$} & \multicolumn{2}{|c|}{$\begin{array}{c}\text { Effectifs (Contextes } \\
\text { d'élision / Total des } \\
\text { contextes) }\end{array}$} & \multicolumn{2}{|c|}{ Taux d'élision } \\
\hline & & Le article & Le pronom & Le article & Le pronom \\
\hline \multirow{3}{*}{$\mathrm{T} 1$} & Salomé & $3 / 93$ & $2 / 5$ & $3,2 \%$ & $20 \%$ \\
\hline & Parents de Salomé (DAE) & $51 / 211$ & $28 / 40$ & $24,2 \%$ & $70 \%$ \\
\hline & Parents de Salomé (DAA) & $32 / 67$ & $11 / 13$ & $47,8 \%$ & $84,6 \%$ \\
\hline \multirow{3}{*}{$\mathrm{T} 2$} & Salomé & 29/99 & $14 / 20$ & $29,3 \%$ & $70 \%$ \\
\hline & Parents de Salomé (DAE) & $80 / 165$ & $23 / 30$ & $48,5 \%$ & $76,7 \%$ \\
\hline & Parents de Salomé (DAA) & $29 / 45$ & $2 / 3$ & $64,4 \%$ & $66,7 \%$ \\
\hline \multirow{3}{*}{$\mathrm{T} 1$} & Baptiste & $1 / 85$ & $0 / 1$ & $1,2 \%$ & $0,0 \%$ \\
\hline & $\begin{array}{c}\text { Parents de Baptiste } \\
\text { (DAE) }\end{array}$ & 23/137 & $14 / 22$ & $16,8 \%$ & $63,6 \%$ \\
\hline & $\begin{array}{l}\text { Parents de Baptiste } \\
\text { (DAA) }\end{array}$ & $18 / 30$ & $6 / 8$ & $60,0 \%$ & $75,0 \%$ \\
\hline \multirow{3}{*}{$\mathrm{T} 2$} & Baptiste & $9 / 67$ & $0 / 3$ & $13,4 \%$ & $0,0 \%$ \\
\hline & $\begin{array}{c}\text { Parents de Baptiste } \\
\text { (DAE) }\end{array}$ & $20 / 85$ & 9/13 & $23,5 \%$ & $69,2 \%$ \\
\hline & $\begin{array}{c}\text { Parents de Baptiste } \\
\text { (DAA) }\end{array}$ & $38 / 91$ & $6 / 12$ & $41,8 \%$ & $50,0 \%$ \\
\hline \multirow{3}{*}{$\mathrm{T} 1$} & Prune & $34 / 130$ & $32 / 66$ & $26,2 \%$ & $48,5 \%$ \\
\hline & Parents de Prune (DAE) & 27/115 & $15 / 40$ & $23,5 \%$ & $37,5 \%$ \\
\hline & Parents de Prune (DAA) & $19 / 31$ & $6 / 7$ & $61,3 \%$ & $85,7 \%$ \\
\hline \multirow{3}{*}{$\mathrm{T} 2$} & Prune & $29 / 65$ & $7 / 7$ & $44,6 \%$ & $100 \%$ \\
\hline & Parents de Prune (DAE) & $15 / 37$ & $13 / 20$ & $40,5 \%$ & $65 \%$ \\
\hline & Parents de Prune (DAA) & $6 / 9$ & $0 / 1$ & $66,7 \%$ & $0 \%$ \\
\hline
\end{tabular}

Tableau 4 : Taux et contextes d'élision du schwa dans le monosyllabe «le »

L'observation du Tableau 4 nous permet de voir que l'emploi du «le » en tant qu'article est plus fréquent dans le discours parental comme dans le discours enfantin. De plus, le nombre de productions de «le» en tant que pronom par les parents semblent s'ajuster en fonction du développement linguistique de l'enfant. En effet, les parents de Baptiste sont ceux qui en produisent le moins, alors que l'utilisation du "le » comme pronom par Baptiste reste marginal (seulement 4 contextes relevés en près de 10 heures de conversations enregistrées). Malgré tout, les effectifs dans le discours parental apparaissent assez nombreux pour pouvoir mener des analyses sur son utilisation en fonction de l'adresse du discours.

De façon générale, en excluant les conditions où les effectifs sont trop faibles pour être comparés, l'élision semble toucher davantage le monosyllabe «le employé comme pronom. Cette remarque est valable, dans le discours parental, à la fois en DAA et en DAE. Cependant, la modulation du langage par les parents en fonction de l'adresse du discours parait beaucoup plus importante en contexte nominal qu'en 
contexte verbal. Chez les parents de Prune, par exemple, le taux d'élision du schwa dans «le» employé comme pronom reste relativement élevé, même lorsque ces derniers s'adressent à leur fillette. Ainsi, il apparaît que les parents cherchent à poser une démarcation plus forte, dans la chaine de la parole, entre un article et un nom ou un adjectif qu'entre un pronom et un verbe. Cet effort de clarification de la séparation déterminant/nom dans le flux continu de la parole pourrait ainsi limiter la mémorisation d'un exemplaire d'un substantif issu d'une mauvaise syllabation, comme on peut l'observer dans des contextes de liaison où la consonne de liaison se trouve mémorisée à l'initiale du nom (Chevrot et al., 2009). De plus, nos données révèlent un réel ajustement du discours parental en fonction de la compétence linguistique des enfants. En effet, entre T1 et T2, une augmentation du taux d'élision est perceptible aussi bien dans le discours enfantin que dans le DAE, mais pas nécessairement en DAA. Afin d'approfondir ces résultats sur le groupe nominal, nous avons souhaité observer la variation maintien/élision du schwa en fonction de chaque contexte le + nom/adjectif. Ainsi, nous souhaitons vérifier si les parents offrent à leur enfant, pour un même mot, des contextes le + nom/adjectif stables (avec un schwa systématiquement élidé ou systématiquement variable) ou variables. Pour ce faire, chaque contexte a été annoté en fonction de ces trois critères ${ }^{4}$ :

- contexte systématiquement maintenu : les parents maintiennent systématiquement le schwa devant un mot donné. Par exemple, la suite «le bébé » est toujours réalisée avec un schwa maintenu ;

- contexte systématiquement élidé : les parents élident systématiquement le schwa devant un mot donné. Par exemple, la suite «le bébé » est toujours réalisée avec un schwa élidé ;

- contexte variable: lorsque l'on trouve, dans le corpus, au moins une élision et un maintien du schwa devant un mot donné. Par exemple, la suite « le bébé » est parfois réalisée avec un schwa maintenu, par fois avec un schwa élidé.

Les données issues du DAE révèlent que, au premier temps de récolte des données, les parents privilégient fortement la production systématique du schwa devant un nom ou un adjectif donné (cf. Tableau 5). Ainsi, la grande majorité des mots rencontrés par l'enfant dans la structure le + Nom/Adj. sont systématiquement produits par les parents avec la forme pleine de l'article au T1. Entre T1 et T2, les parents tendent à diversifier les types de contextes produits en direction de l'enfant, dans des proportions sensiblement proches de celles observées dans les productions enfantines (cf. Figures 3 à 5). Ainsi, alors que Salomé et Prune se mettent à produire en T2 davantage de contextes avec une élision systématique du schwa de l'article, la même caractéristique est observable dans le discours parental adressé à l'enfant. Parallèlement, la proportion des contextes impliquant une production systématique du schwa est en baisse dans les productions des deux couples de parents au T2. Baptiste, quant à lui, commence simplement à produire des contextes variables ou systématiquement élidés au T2, dans des proportions beaucoup plus faibles que Salomé et Prune. Cette caractéristique des productions de Baptiste se manifeste

${ }^{4}$ Nous remercions Laurine Ségur de son aide pour l'annotation des données. 
également dans le DAE : les proportions des contextes restent stables entre T1 et T2, les contextes où le schwa est systématiquement maintenu dominant toujours aussi nettement au T2.

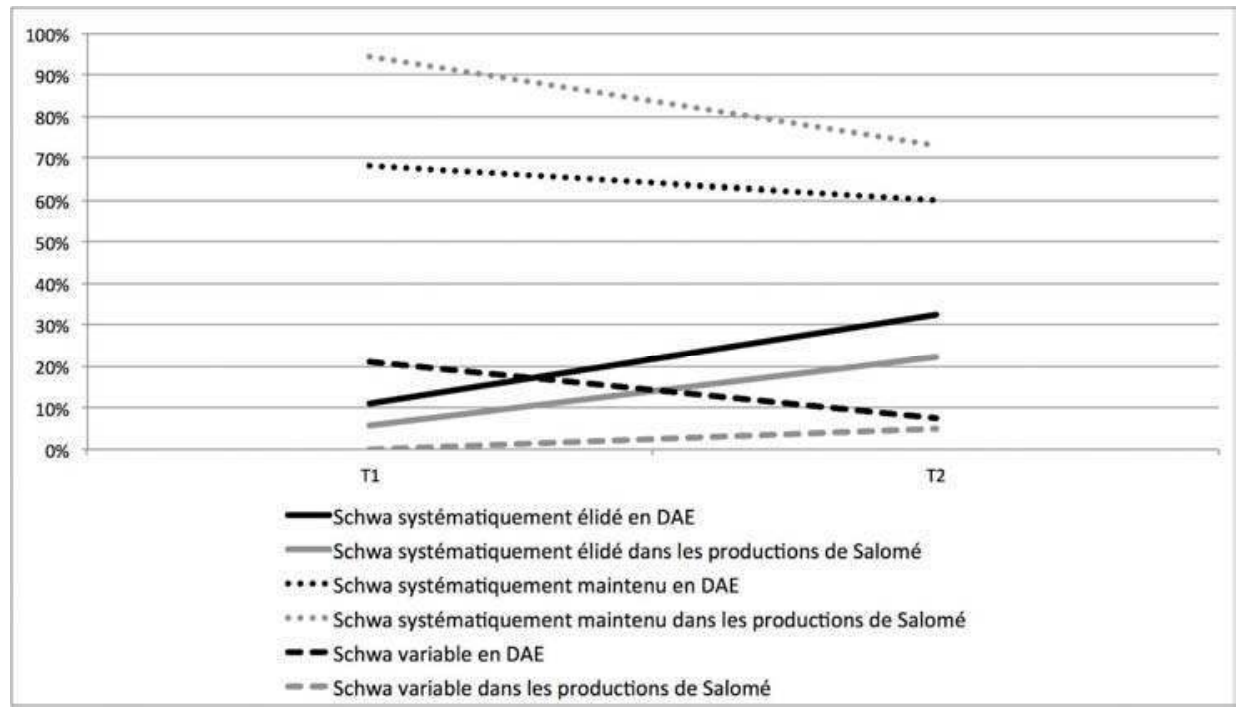

Figure 3 :

Systématicité et variation dans l'élision et le maintien du schwa dans les productions de Salomé et de ses parents

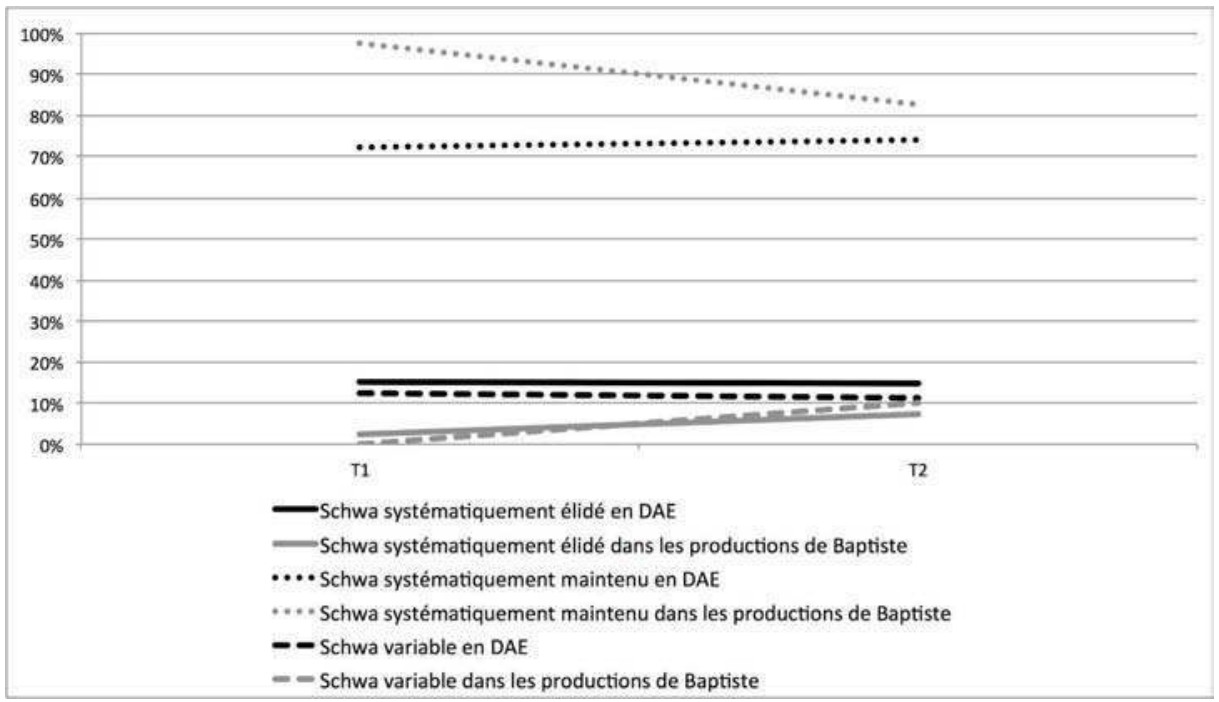

Figure 4 :

Systématicité et variation dans l'élision et le maintien du schwa dans les productions de Baptiste et de ses parents 


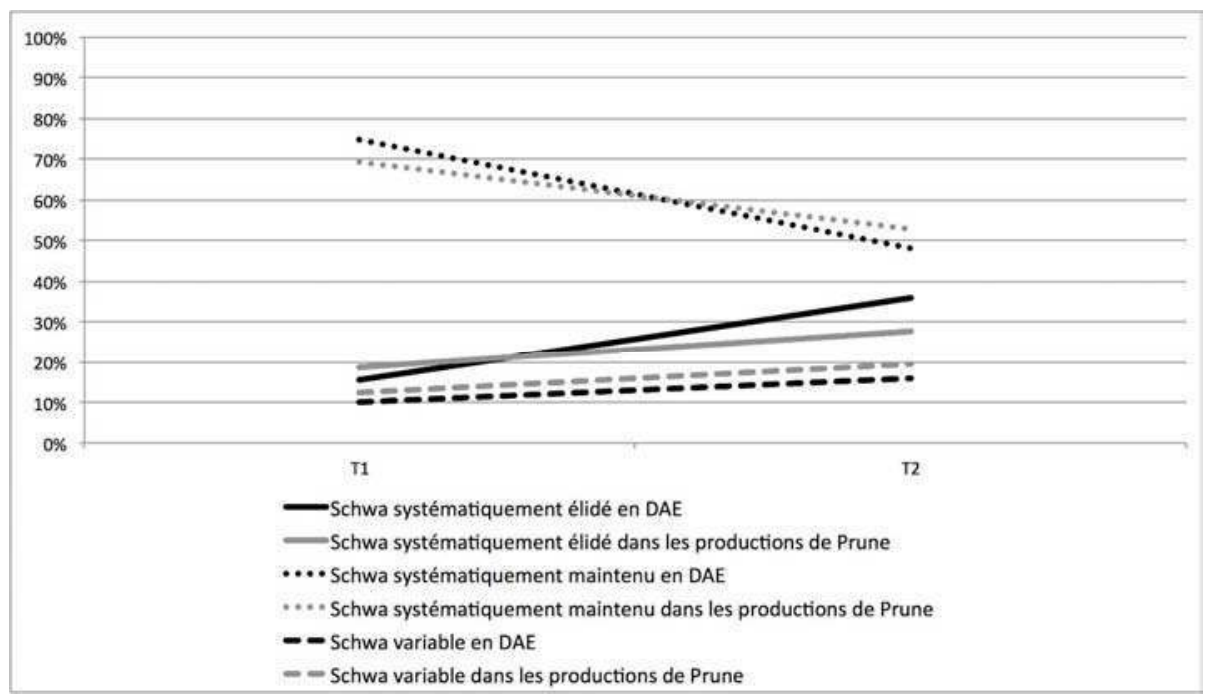

Figure 5 :

Systématicité et variation dans l'élision et le maintien du schwa dans les productions de Prune et de ses parents

\begin{tabular}{|c|c|c|c|c|c|c|}
\hline \multirow{2}{*}{ Type de contexte } & \multicolumn{2}{|c|}{ Parents de Salomé } & \multicolumn{2}{c|}{ Parents de Baptiste } & \multicolumn{2}{c|}{ Parents de Prune } \\
\cline { 2 - 7 } & $\mathrm{T} 1$ & $\mathrm{~T} 2$ & $\mathrm{~T} 1$ & $\mathrm{~T} 2$ & $\mathrm{~T} 1$ & $\mathrm{~T} 2$ \\
\hline $\begin{array}{c}\text { Schwa } \\
\text { systématiquement élidé }\end{array}$ & $11 \%$ & $32,5 \%$ & $15,3 \%$ & $14,8 \%$ & $15,5 \%$ & $36 \%$ \\
\hline $\begin{array}{c}\text { Schwa } \\
\text { systématiquement } \\
\text { maintenu }\end{array}$ & $68 \%$ & $60 \%$ & $72,2 \%$ & $74,1 \%$ & $74,6 \%$ & $48 \%$ \\
\hline Schwa variable & $21 \%$ & $7,5 \%$ & $12,5 \%$ & $11,1 \%$ & $9,9 \%$ & $16 \%$ \\
\hline
\end{tabular}

Tableau 5 : Systématicité et variation dans l'élision et le maintien du schwa

\section{Conclusions : caractéristiques de l'input et acquisition de l'élision}

Ce travail avait un double objectif. Dans un premier temps, nous souhaitions étendre les résultats obtenus dans nos précédents travaux (Liégeois et al., 2012) à un troisième sujet dans le but d'observer si ses parents modulaient leur usage su schwa en fonction de l'adresse du discours (discours adressé à l'adulte ou à l'enfant). L'analyse des productions des parents de Salomé, Baptiste et Prune nous révèlent que les adultes ont tendance à ajuster leur réalisation du schwa lorsqu'ils s'adressent à leur enfant. En effet, nous notons chez les trois couples de parents une différence très significative entre les taux d'élision en DAE et en DAA lors du premier temps de recueil. Ainsi, comparé au DAA, le DAE comporte bien des spécificités au niveau de la variation phonologique. À un stade précoce du développement, les enfants sont exposés majoritairement aux formes standard des monosyllabes avec un schwa maintenu. Entre T1 et T2, le taux d'élision en DAE augmente significativement chez les parents 
dont l'enfant se met à élider le schwa dans des proportions relativement proches aux leurs (Salomé et Prune). Dans le même temps, alors que Baptiste a toujours tendance à nettement privilégier la forme pleine des monosyllabes au T2 (seulement 20\% d'élision environ), nous ne relevons pas de différence significative entre T1 et T2 dans les taux d'élision relevés chez ses parents en DAE. Ainsi ces derniers adoptent, comme au T1, un comportement différent vis-à-vis de la variation phonologique en fonction de l'adresse de leur discours. Le schwa est davantage maintenu en DAE, dans des proportions significativement plus élevées comparées à celles relevées en DAA au même temps de recueil. Nous interprétons ces observations comme un ajustement des caractéristiques phonologiques du discours en fonction des compétences de l'enfant. À un stade précoce, le maintien majoritaire du schwa permettrait de maximiser l'intelligibilité des énoncés produits. En effet, l'enfant se basant sur des schémas phonologiques récurrents pour segmenter les énoncés, l'input répondrait au schéma classique de la syllabe en français, la structure Consonne-Voyelle. Ainsi, comme Racine (2007) l'a montré pour les substantifs polysyllabes comportant un schwa en syllabe initiale, il serait intéressant de vérifier à l'aide de procédures expérimentales si les enfants préfèrent et accèdent plus rapidement au mot suivant le monosyllabe si le schwa est maintenu dans celui-ci.

Le deuxième objectif de cette recherche était de vérifier si, comme Andreassen (2011) l'a démontré pour le contexte V\#Cə, les parents modulent leur discours en fonction du contexte syntaxique pour les monosyllabes. Nous nous sommes ainsi focalisés sur le monosyllabe «le» en distinguant son usage en tant qu'article précédant un nom ou un adjectif et en tant que pronom précédant une forme verbale. Nous avons observé une nette différence de taux d'élision entre les deux contextes d'emploi du monosyllabique «le». Dans les productions des parents de nos trois sujets, nous avons relevé une modulation en fonction de l'adresse du discours beaucoup plus forte en contexte nominal qu'en contexte verbal. Nous formulons donc l'hypothèse qu'aux premiers stades de l'acquisition du schwa, les parents, en modulant leur discours, se focaliseraient davantage sur le vocabulaire et les aspects concrets et référentiels du langage. En réalisant le schwa, ils chercheraient à faciliter l'analyse et la syllabisation des mots prononcés afin que l'enfant accède à toutes les composantes du groupe nominal et acquière plus rapidement le lexique proposé. Par ailleurs, lorsqu'il s'agit du contexte «le $+V e r b e »$, les parents ne feraient pas le même effort d'analyse et de simplification, ce qui expliquerait en partie le taux d'élision élevé au sein de ce contexte syntaxique. En ce qui concerne les productions enfantines, nous avons observé qu’à un stade précoce de l'acquisition, l'élision du schwa dans le monosyllabe «le »employé comme article est marginale $(3,2 \%$ chez Salomé à $2 ; 4$ ans, $1,2 \%$ chez Baptiste à $3 ; 0$ ans). Dans le même temps, les enfants semblent exposés à une variation très limitée dans l'input, les parents employant en majeure partie systématiquement la forme pleine de l'article avant un nom ou un adjectif particulier. Cet état de fait pourrait provenir de la lecture d'histoires aux enfants générant une lecture fréquente et normée des contextes «le $+\mathrm{Nom} / A d j$. » et des questions permettant de mettre en relief ces formes. Maratsos (1976) a réalisé une étude sur l'article défini «the» et l'indéfini « $a$ » avec des enfants de 36 à 48 mois. L'auteur a montré une production plus conséquente de l'article «the » par rapport à «a». Selon lui, cela serait dû à la lecture fréquente 
d'histoires par les parents et au «storytelling», un moment où le récit relaté à l'enfant est ponctué de dénominations, de questions sur l'animal, le personnage ou l'objet illustré dans le support écrit. Ces actions répétées en situation de lecture avec une mise en relief du «le » dans des questions du type «que fait le $X$ ?, où est le $X$ ?... » pourraient être à l'origine d'une meilleure articulation du «le» avec la présence du schwa dans l'input parental.

\section{Références}

ADREASSEN, H. (2011), « La recherche de régularités distributionnelles pour la catégorisation du schwa en français », Langue française, 169, p. 55-78.

Barlow, M. \& Kemmer, S. (éds.) (2000), Usage-based Models of Language, Stanford, Californie, CSLI Publications.

BORNSTEIN, M., HAHN, C.-S. \& HAYNES, O. (2004), « Specific and general language performance across early childhood: Stability and gender considerations », First Language, 24, p. 267-304.

Behrens, H. (éd.) (2008a), Corpora in Language Acquisition Research: History, Methods, Perspectives. Amsterdam, John Benjamins.

BEHRENS, H. (éd.) (2008b), « Corpora in language acquisition research. History, methods, perspectives », dans H. Behrens (éd.), Corpora in Language Acquisition Research: History, Methods, Perspectives. Amsterdam, John Benjamins, p. XI-XXX.

Bürki, A, Racine, I., Adreassen, H., Fougeron, C. \& Frauenfelder, U. (2008), « Timbre du schwa en français et variation régionale : une étude comparative», Actes des journées d'étude sur la parole, Avignon.

Bybee, J. \& Hopper, P. (éds.) (2001), Frequency and the Emergence of Linguistic Structure, Amsterdam, John Benjamins.

BybeE, J. (2010), Language, Usage and Cognition, Cambridge, Cambridge University Press.

CHABANAL, D. (2003) : Un aspect de l'acquisition du français oral: la variation sociophonétique chez l'enfant francophone, thèse de doctorat, Université Paul Valéry - Montpellier 3.

Chabanal, D. \& Durand, J. (2015). «French in Auvergne (Centre): a speaker from ClermontFerrand» in S. Detey, J. Durand, B. Laks, C. Lyche (éds.), Varieties of Spoken French, Oxford, Oxford University Press, p. 5-15.

CHENU, F. \& JISA, H. (2005) : «Impact du discours adressé à l'enfant sur l'acquisition des verbes en français », Revue de linguistique et de didactique des langues, 31, p. 85-100.

Chevrot, J.-P. \& FAyol, M. (2001), « Acquisition of French liaison and related child errors », in M. Almgren, A. Barreña, M. J. Ezeizabarena, I. Idiazabal \& B. MacWhinney (éds.), Research on Child Language Acquisition: Proceedings of the 8th Conference of the International Association for the Study of Child Language, Somerville, Cascadilla Press, p. 760-774.

Chevrot, J.-P., Dugua, C. \& FAYOL, M. (2009), «Liaison acquisition, word segmentation and construction in French: a usage based account », Journal of child Language, 36/3, p. 557-596. 
DARWIN, C. (1877), « A biographical sketch of an infant », Mind, 2/7, p. 285-294.

DeLAIS-RoussariE, E. (2004), «Constitution et annotation de corpus : méthode et recommandations », dans E. Delais-Roussarie \& J. Durand (éds.), Corpus et variation en phonologie : méthodes et analyses, Toulouse, Presses Universitaires du Mirail, p. 89-126.

DeLL, F. (1973), Les règles et les sons. Paris, Hermann.

DeLl, F. (1978), «Epenthèse et effacement de schwa dans des syllabes contiguës en français », dans B. de Cornulier \& F. Dell (éds.), Études de phonologie française, Paris, Éditions du CNRS, p. 75-81.

Dilley, L., Millett, A. MCauley, J \& Bergeson, T. (2013), « Phonetic variation in consonants in infant-directed and adult-directed speech: the case of regressive place assimilation in word-final alveolar stops », Journal of Child Language, 41/1, p. 155-175.

DuRAND, J., LAKS, B. \& C. LYCHE (2002), «La phonologie du français contemporain: usages, variétés et structure », dans C. Pusch \& W. Raible (éds.), Romanistische Korpuslinguistik Korpora und gesprochene Sprache/Romance corpus linguistics - Corpora and spoken language, Tubingue, Gunter Narr Verlag, p. 93-106.

DurAND, J., LAKS, B. \& C. LYCHE (2009), «Le projet PFC (Phonologie du Français Contemporain) : une source de données primaires structurées », dans J. Durand, B. Laks \& C. Lyche (éds.), Phonologie, variation et accents du français, Paris, Hermès, p. 19-61.

DugUA, C. (2006), Liaison, segmentation lexicale et schémas syntaxiques entre 2 et 6 ans. Un modèle développemental basé sur l'usage, thèse de doctorat, Université Stendhal - Grenoble III.

EyCHENNE, J. (2006), Aspects de la phonologie du schwa dans le français contemporain. Optimalité, visibilité prosodique, gradience, thèse de doctorat, Université de Toulouse - Le Mirail.

Foulkes, P., Docherty, G. \& WATT, D. (2005), «Phonological variation in child-directed speech », Language, 81/1, p. 177-206.

GARNICA, O. (1977), «Some prosodic and paralinguistic features of speech to young children », dans C. Snow \& C. Ferguson (éds.), Talking to Children: Language Input and Acquisition, Cambridge, Cambridge University Press, p. 63-88.

GENOUD, P. (2011), «Indice de position socioéconomique (IPSE) : un calcul simplifié ». Fribourg, Université de Fribourg, www.unifr.ch/ipg/assets/files/DocGenoud/IPSE.pdf.

GoldberG, A. (2006), Constructions at Work: The Nature of Generalization in Language, Oxford, Oxford University Press.

Huttenlocher, J., HAight, W. BryK, A. \& Seltzer, M. (1991), « Early vocabulary growth: relation to language input and gender », Developmental Psychology, 27/2, p. 236-248.

JisA, H. \& Richaud, F. (1994), "Quelques sources de variation chez les enfants », AILE, 4, p. 367-376.

LAKS, B. (2011), « La phonologie du français et les corpus », Langue française, 169, p. 3-17.

LIÉGEOIS, L. (2013), «De l’analyse au partage des données, quel(s) format(s) choisir ? L'exemple d'un corpus d'interactions parents-enfant", dans M. Damiani, K. Dolar, C. FlorezPulido, R. Loth, J. Magnier, \& A. Pegaz (éds.), Traitement de corpus, Actes de Coldoc 2012. Paris, Modyco, p.128-142. 
LiÉgeois, L., ChaBAnal, D. \& CHAnier, T. (2011), « La liaison en discours adressé à l'enfant, spécificités et impacts sur l'acquisition », Colloque du résean français de phonologie, Tours, 1-3 juillet 2011.

LiÉGeois, L., Chanier, T. \& Chabanal, D. (2014), «Corpus globaux ALIPE : Interactions parents-enfant annotées pour l'étude de la liaison », Nancy, Ortolang, http://hdl.handle. net/11041/alipe-000853/.

LiÉGEOIS, L., SADDOUR, I. \& CHABANAL, D. (2012), «L'élision du schwa dans les interactions parents-enfant : étude de corpus ", dans L. Besacier, B. Lecouteux \& G. Sérasset (éds.), Actes de la conférence conjointe JEP-TALN-RECITAL 2012, Grenoble, ATALA \& AFCP, p. 313-320.

MARATSOS, M. (1976), The use of definite and indefinite reference in young children, Cambridge, Cambridge University Press.

MACWhinneY, B. \& SNOW, C. (1985), «The child language data exchange system », Journal of Child Language, 12/2, p. 271-295.

MaCWHINnEY, B. (2000), The CHILDES Project: Tools for Analyzing Talk, Mahwah NJ, Lawrence Erlbaum.

Martinet, A. (1972), «La nature phonologique d'e caduc», dans A. Valdman (éd.), Papers in Linguistics and Phonetics to the Memory of Pierre Delattre, La Haye, Mouton, p. 393-399.

Nicoladis, E. \& PARADIS, J. (2011), «Learning to liaise and elide comme il faut. Evidence from bilingual children », Journal of Child Language, 38/4, p. 701-730.

PArisse, C. \& Morgenstern, A. (2010a), «Transcrire et analyser les corpus d'interactions adulte-enfant », dans E. Veneziano, A. Salazar \& J. Bernicot (éds.), Acquisition du langage et interaction, Paris, L'Harmattan, p. 201-222.

PARISSE, C. \& MORGENSTERn, A. (2010b), « A multi-software integration platform and support for multimedia transcripts of language », LREC 2010, Workshop on Multimodal Corpora: Advances in Capturing, Coding and Analyzing Multimodality, La Valette, Malte, p.106-110.

Pierrehumbert, J. (2001), «Exemplar dynamics: Word frequency, lenition and contrast », in J. Bybee \& P. Hopper (éds.), Frequency Effects and the Emergence of Lexical Structure, Amsterdam, John Benjamins, p. 137-158.

RACINE, I. (2007), «Effacement du schwa et reconnaissance des mots chez les enfants », Actes des JEL 2007, p. 119-124.

RONDAL, J. (1980), «Fathers' and mothers' speech in early language development », Journal of Child Language 7/2, p. 353-369.

SCHANE, S. (1968), «On the abstract character on French e muet », Glossa, 2, p. 150-163.

SNOw, C. (1972), «Mothers' speech to children learning language », Child Development, 43/2, p. $549-565$.

TAINE, M. (1876), «L'acquisition du langage chez les enfants et dans l'espèce humaine », Revue philosophique de la France et de l'étranger, 1, p. 357-395.

TAINE, M. (1877), « M. Taine on the acquisition of language by children », Mind, 2/6, p. 252-259. 
Tomasello, M. (2003), Constructing a Language: A Usage-based Theory of Language Acquisition, Cambridge MA, Harvard University Press.

TOMASELLO, M., \& STAHL, D. (2004), « Sampling children's spontaneous speech: how much is enough? », Journal of Child Language, 31/1, p. 101-121.

Uther, M., Knoll M. \& Burnham, D. (2007), « Do you speak E-NG-L-I-S-H? A comparison of foreigner- and infant-directed speech », Speech Communication, 17, p. 2-7.

Wall, L., Christiansen, T. \& Orwant, J. (2000/2001, traduction), Programmation en Perl, Paris, O’Reilly. 


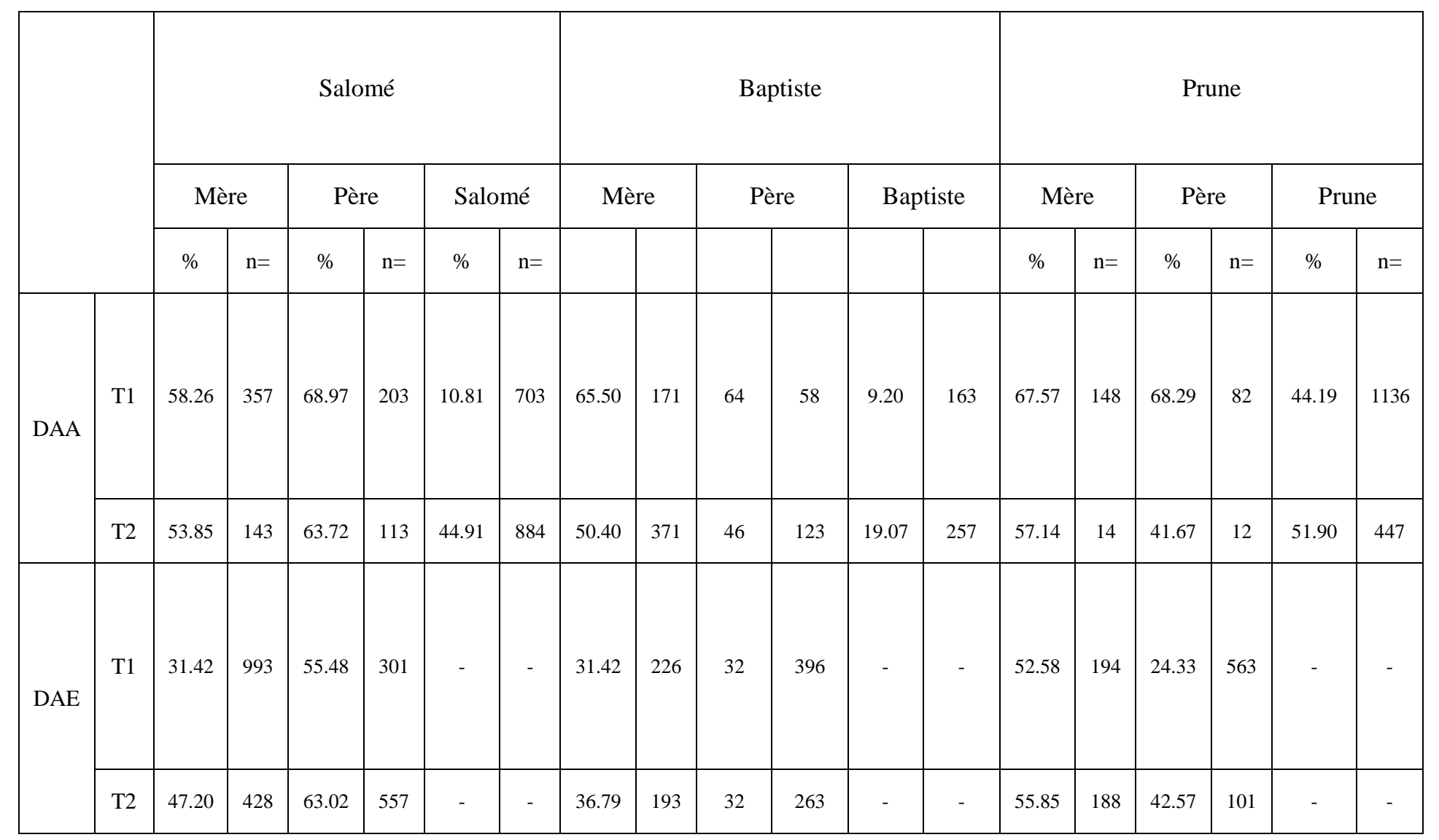

68 